\title{
Diagnosis and Management of Post-Stroke Fatigue: What Do We Know, What Do We Still Need To Know?
}

\author{
Benjamin Tseng ${ }^{1 *}$ and Colleen Marzilli ${ }^{2}$ \\ ${ }^{1}$ Department of Health and Kinesiology, USA \\ ${ }^{2}$ School of Nursing, USA
}

*Corresponding author: Benjamin Tseng, Laboratory of Brain Aging and Neuromotor Behavior, Department of Health and Kinesiology Submission: 海 March 19, 2018; Published: 海 April 11, 2018

\begin{abstract}
Although Post-stroke fatigue (PSF) has gained attention in recent years, clinicians and caregivers are still faced with the challenge of diagnosis and management of PSF. The purpose of this review is to provide understanding of the nature of PSF and potential clinical implication for healthcare providers.
\end{abstract}

Keywords: Stroke; Stroke care; Post stroke fatigue

\section{Editorial}

\section{The classical view of post-stroke fatigue}

Post-stroke fatigue (PSF) occurs in more than half of stroke survivors around the world [1] and has been linked with poor quality of life, independent of depression and disability $[2,3]$. PSF has a profound negative impact on a patient's daily activities such as decreased participation in physical activities [4], leading to poor rehabilitation outcome and increased mortality [5]. Previous literature that examined other clinical conditions defines fatigue as a subject feeling of physical and/or mental exhaustion, lack of energy, and tiredness perceived by the individual or caregiver. To date, consensus on one definition of PSF has yet to be reached among clinicians and researchers.

\section{What's the challenge?}

The debilitating effects of PSF and its hindrance on stroke rehabilitation [4] creates dire consequences on the quality of life in people after stroke [5]. Although PSF has gained attention in recent years, clinicians and caregivers are still faced with the challenge of diagnosis and management of PSF. Because of the complex and ambiguous nature of PSF, it is often under-diagnosed and under-treated. Current standard of care for chronic stroke relies on educational materials and does not incorporate any proactive countermeasure to alleviate PSF symptoms. Currently, no pharmacological intervention can effectively improve PSF symptoms [6]; while non- pharmacological intervention strategy is a complete void.

\section{The construct of PSF}

The pathophysiology of PSF is largely unknown although some potential factors have been suggested include changes in neurophysiology after stroke such as white matter hyperintensities and inflammation. Accumulating evidence suggests that the cause of PSF is multidimensional $[7,8]$ attributing to the interactions between neurophysiologic anomalies, physical deficits, comorbidities, medications, and psychological burden such as depression, anxiety, and lack of social interactions.

Furthermore, it is important to recognize that PSF has different subtypes that may co-exist simultaneously [7]. Namely, exertion (ePSF) and chronic (cPSF) fatigue subtypes have been identified as 2 distinct PSF constructs each with its own unique contributing factor [7].

\section{Physical impairment and motor dysfunction}

Physical impairment and functional deficits are one of the most observable culprits contributing to PSF symptoms, especially those who exhibit ePSF. The importance of these contributing factors to PSF has been well established [9,10]. Motor dysfunction [11,12], aphasia [4,5], facial palsy [8], and arm weakness [8] are all related to PSF. However, investigations of the impact of the multiple diverse deficits on PSF are scarce.

\section{Depression, anxiety, and social interactions}

Although physical disability may contribute to ePSF in the early stage of stroke, psychological burdens such as depression may play 
a more important role in the chronic stage [7]. It may be worthwhile to recognize that the impact of depression on ePSF may differ depends on the severity of stroke. Interestingly, although some patients with cPSF exhibit depression symptoms, there are patients with fatigue who are not depressed $[5,8]$. Of note, pharmacological therapy for depression is ineffective at treating general PSF, although it improves depression [13]. Thus, there are reasons to believe that, although related, cPSF and post-stroke depression are distinct clinical entities.

The link between PSF and anxiety has been reported [5,14,15] although it does not appear to be as strong as the link between PSF and depression [16]. Nevertheless, it has been shown that stroke survivors with PSF have difficulty in resuming social, familial, and professional activities [17].

\section{Brain structural abnormalities}

Over the past two decades, the revolutionary improvement in neuroimaging techniques has enabled investigators to obtain unprecedented insights into the pathophysiology of the stroke brain. White matter hyperintensities (WMH), or leukoaraiosis, a feature of small cerebral vessel pathologies and/or WM fiber micro structural abnormalities revealed by Magnetic Resonance Imaging (MRI) has been shown to have a strong association with stroke [1821]. A longitudinal study that examined chronic fatigue syndrome found that fatigue severity is associated with white matter hyperintensities [22]. Furthermore, sub cortical white matter injury is predictive of 1-year outcome of PSF predict of fatigue in stroke [23]. More importantly, WMH has been independently associated with PSF [14], it also has been demonstrated that WMH volume is predictive of stroke outcome [24].

\section{How can we measure PSF?}

Due to its multidimensional nature, it may be necessary to first determine a PSF phenotype using a multidimensional model before PSF severity level can be adequately measured.

\section{Quantify ePSF severity level using a visual analog scale}

A version of visual analog scale specifically modified for stroke patients was previously described to quantify fatigue level in stroke patients [25]. Namely, the Visual Analog Fatigue Scale (VAFS), which consists of score ranges from 0 to 100 measured in millimeters on a $10-\mathrm{cm}$ vertical line using a pen (Figure 1). The score is obtained by measuring the line from "no fatigue" to the point indicated by participants to represent their fatigue level. Participants are presented the VAFS for the first time to measure fatigue at rest $\left(\mathrm{VAFS}_{\text {at-rest }}\right.$ ) after sitting for 5 minutes in a quiet room. Immediately afterward, a 15-minute standardized fatigue-inducing

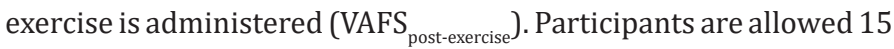
minutes for recovery, after which they will be presented the VAFS for the third time (VAFS $_{\text {post-recovery }}$ ). Exertion fatigue is calculated by subtracting the VAFS $_{\text {at-rest }}$ score from the VAFS ${ }_{\text {post-exercise }}$ score, and recovery rate is calculated using the formula: VAFS $_{\text {post-exercise }}$ - VAFS post-recovery $) /\left(\right.$ VAFS $_{\text {post-exercise }}-V_{\text {VAFS }}$ at-rest $\left.) \times 100\right)$. The fatigueinducing exercise consists of a 15- minute standardized exercise protocol using a total body recumbent stepper (NuStep, Inc). To standardize the workload, all participants are asked to step at 75 steps per minute with an external power of 75 to 80 watts for 15 minutes. The device and workload of the fatigue-inducing protocol was previously validated $[7,25]$.

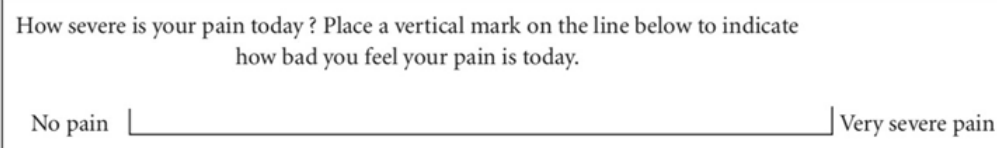

(a)

Visual Analog Fatigue Scale (VAFS)

Visual Analog Fatigue Scale (VAFS)

At rest — Post exercise — Post recovery — At rest — Post exercise — P P recovery _ - P

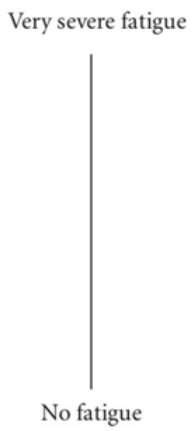

(b)

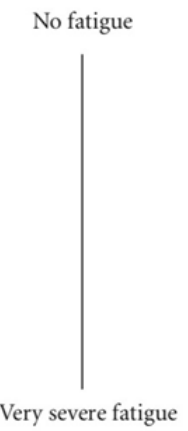

(c)

Figure 1: Illustration of (a) traditional visual analog scale used to measure pain, (b) VAFS version 1, and (c) VAFS version 2. VAFS: Visual Analog Fatigue Scale. 
The VAFS itself, the assessment of ePSF using the change score on the VAFS, and the calculation of recovery rate using the ratio of VAFS change score have been established previously and reported to have good validity in stroke survivors [25].

\section{Quantify cPSF severity level using the fatigue severity scale}

The Fatigue Severity Scale (FSS) [26] is a 9-item scale that has been shown to be reliable and valid to assess fatigue level over the previous week in stroke survivors. It can be utilized to quantify the chronic subtype of PSF (cPSF). In addition, several other fatigue scales are also suitable to assess CPSF such as the Modified Fatigue Impact Scale (MFIS) and the Multidimensional Fatigue Symptom Inventory (MFSI).

Physical fitness \& function: Physical function should be evaluated using the 6-minute walk, Fugl-Meyer (FM) tests. The FM is used to determine the level of motor function in the hemiparetic limbs after stroke. The FM test is a reliable and valid tool that was specifically designed as a clinical measure of sensori- motor impairment for stroke [27].

The total possible score is 124 , which has components of sensation (FMSEN, 24 points), upper extremity (FMUE, 66 points), and lower extremity (FMLE, 34 points). Because the sensory component is less relevant, the combined total motor (FMTM) scores of the FMUE and FMLE can be determined as (FMTM=FMUE+FMLE) [28], with a total possible score of 100. The 6-minute walk test is a field assessment of aerobic fitness.

Psychological burden: Depressive symptoms, anxiety level, and social interactions should be evaluated using the Geriatric Depression Scale (GDS), Penn State Worry Questionnaire (PSWQ), and Older People's Quality of Life Questionnaire (OPQoL), respectively. The GDS is a questionnaire that includes 30 items that refer to affective, cognitive, and behavioral symptoms of depression to assess mood. It has been tested extensively in older adults and previously used in stroke survivors $[7,25]$. The possible score ranges from 0 to 30 . The PSWQ is a widely used measure of worry severity [29]. The OPQoL is a new 32- to 35-item QoL measure [30]. It has 5-point Likert scales from Strongly Agree to Strongly Disagree, with 32 or 35 items, representing: life overall (4 items), health (4 items), social relationships and participation (7 items in QoL follow-up survey, 8 items in Omnibus surveys), independence, control over life, freedom (5 items), area: home and neighborhood (4 items), psychological and emotional well- being (4 items), financial circumstances (4 items), and religion/culture (2 items; asked in Omnibus surveys only).

\section{Healthcare provider implications}

The diagnosis of stroke is one that brings about great change for not only the patient, but the family, too. However, that does not consider the effect of PSF on the patient and the implications for the family.
Following stroke, even without any permanent disabilities, the family often experiences guilt. Families often ask, "Did that ice cream for dessert cause the stroke?" This is only the tip of the iceberg describing the thoughts that the families of those with stroke experience. The patient is often racked with guilt over subjecting the family to this life-changing health condition. Patients with stroke are more likely to experience depression and anxiety related to their decline in health and possible need for assistance and permanent lifestyle modifications. When post-stroke fatigue is present, these feelings of guilt, depression, and anxiety are often magnified. When PSF is present, the healthcare provider can support the patient and family by addressing activity and sleep, diet, and mental health. The health care provider should consider activity and a healthy sleep schedule. The level of activity should be tailored to the patient's individual needs including pre-stroke level of functioning and consider any residuals following the stroke. The family should be encouraged to participate in the activity level with the patient and help support the patient through a custom-tailored activity plan. Activities can be as simple as playing a card game to things like going for a walk or on a hike. Bird-watching and woodworking are examples of activities that the patient with post-stroke fatigue might find enjoyable through the process of managing poststroke fatigue. The healthcare provider should counsel the patient on appropriate sleep hygiene and encourage a healthy sleep/wake routine. A scheduled bedtime with appropriate number of hours of sleep followed by a standard wake time with limited naps are helpful in managing post-stroke fatigue. The health care provider should council against indulging in too much sleep as this is counter-productive to managing post-stroke fatigue.

Two, diet and nutrition are essential for the healthcare provider to address following a stroke, and when post-stroke fatigue is present, this is essential to help manage the fatigue. The healthcare provider should consider a nutritional consult, and provide dietary resources such as websites for healthy food preparation and recipes. Managing diet and nutrition also includes educating the patient about the use of caffeine and stimulants to combat the post-stroke fatigue as these are counterproductive to successfully managing the PSF and consumption can cause other health-related issues.

Three, it is paramount that the healthcare provider support the patient through post-stroke fatigue by addressing mental health such as any anxiety or depression that the patient may experience. Depression often occurs due to a decrease in function and/or quality of life and guilt related to lifestyle decisions and health behaviors that may have contributed to the initial stroke and the patient may experience anxiety with the realization that the chances of another ischemic event are likely. When PSF is present, these conditions can be magnified. The healthcare provider should screen for these conditions and provide referrals and treatment as needed. Medications and mental health care including counseling services may be indicated. Engaging in activity and consuming a healthy diet can also help address these. 
The healthcare provider not only can take on the role of partner in addressing PSF and consider activity and sleep, diet, and mental health for the patient and their family, they also play a key role in supporting the patient and family and really empowering through the process.

\section{What's next?}

Currently there is no evidence for effective pharmacological interventions in patients with PSF. On the other hand, nonpharmacological PSF intervention is inadequate and offers only general education materials and advice patients and caregivers for dealing with PSF.

The immediate urgency of advancing PSF diagnosis should rely on developing a PSF phenotype; while the future PSF management must focus on a multidimensional treatment strategy that is personalized to each individual patient's PSF contributing factors. It is also important to recognize that the healthcare provider is a great resource and help for the patient with post-stroke fatigue and can offer many strategies to combat the effects. Ultimately, this line of research should continue to strive to improve the quality of life in people after stroke as well as reducing the psychological burden of family and caregivers.

\section{Disclosures}

None.

\section{References}

1. Brodtmann A, van de Port IG (2013) Fitness, depression, and post-stroke fatigue: worn out or weary? Neurology 81(18): 1566-1567.

2. Tang WK, Lu JY, Chen YK, Mok VC, Ungvari GS, et al. (2010) Is fatigue associate with short-term health-related quality of life in stroke? Arch Phys Med Rehabil 91(10): 1511-1515.

3. Van de Port IG, Kwakkel G, Schepers VP, Heinemans CT, Lindeman E (2007) Is fatigue an independent factor associated with activities of daily living, instrumental activities of daily living and health-related quality of life in chronic stroke? Cerebrovasc Dis 23(1): 40-45.

4. Choi-Kwon S, Han SW, Kwon SU, Kim JS (2005) Post-stroke fatigue: characteristics and related factors. Cerebrovasc Dis 19(2): 84-90.

5. Glader EL, Stegmayr B, Asplund K (2002) Post-stroke fatigue: a 2-year follow- up study of stroke patients in Sweden. Stroke 33(5): 1327-1333.

6. Poulsen MB, Damgaard B, Zerahn B, Overgaard K, Rasmussen RS (2015) Moda nil may alleviate post-stroke fatigue: a randomized, placebo-controlled, double-blinded trial. Stroke 46(12): 3470-3477.

7. Tseng BY, Billinger SA, Gajewski BJ, Kluding PM (2010) Exertion fatigue and chronic fatigue are two distinct constructs in people post-stroke. Stroke 41(12): 2908-2912.

8. Cumming TB, Packer M, Kramer SF, English C (2016) The prevalence of fatigue after stroke: a systematic review and meta-analysis. Int J Stroke 11(9): 968-977.

9. Appelros P (2006) Prevalence and predictors of pain and fatigue after stroke: a population based study. Int J Rehabil Res 29(4): 329-333.

10. Christensen D, Johnsen SP, Watt T, Harder I, Kirkevold M, et al. (2008) Dimensions of post-stroke fatigue: a two-year follow-up study. Cerebrovasc Dis 26(2): 134-141
11.Van der Werf SP, Van den Broek HL, Anten HW, Bleijenberg G (2001) Experience of severe fatigue long after stroke and its relation to depressive symptoms and disease characteristics. Eur Neurol 45(1): 28-33.

12. Tseng BY, Kluding P (2009) The relationship between fatigue, aerobic tness, and motor control in people with chronic stroke: a pilot study. J Geriatr Phys Ther 32(3): 97- 102.

13. Choi-Kwon S, Choi J, Kwon SU, Kang DW, Kim JS (2007) Fluoxetine is not effective in the treatment of post-stroke fatigue: a double-blind, placebocontrolled study. Cerebrovasc Dis 23(2-3): 103-108.

14. Naess H, Lunde L, Brogger J, Waje-Andreassen U (2012) Fatigue among stroke patients on long-term follow-up. The bergen stroke study. J Neurol Sci 312(1-2): 138-141.

15. Radman N, Staub F, Aboulafia-Brakha T, Berney A, Bogousslavsky J, et al. (2012) Post-stroke fatigue following minor infarcts: A prospective study. Neurology 79(14): 1422-1427

16. Wu S, Barugh A, Macleod M, Mead G (2014) Psychological associations of post-stroke fatigue: A systematic review and meta-analysis. Stroke 45(6): 1778-1783.

17. Staub F, Bogousslavsky J (2001) Fatigue after stroke: a major but neglected issue. Cerebrovasc Dis 12(2): 75-81.

18. Vermeer SE, Hollander M, van Dijk EJ, Hofman A, Koudstaal PJ, et al. (2003) Silent brain infarcts and white matter lesions increase stroke risk in the general population: The rotterdam scan study. Stroke 34(5): 1126-1129.

19. Jeerakathil T, Wolf PA, Beiser A, Massaro J, Seshadri S, et al. (2004) Stroke risk profile predicts white matter hyper intensity volume: The framingham study. Stroke 35(8): 1857-1861.

20. Longstreth WT, Arnold AM, Beauchamp NJ, Manolio TA, Lefkowitz D, et al. (2005) Incidence, manifestations, and predictors of worsening white matter on serial cranial magnetic resonance imaging in the elderly: The cardiovascular health study. Stroke 36(1): 56-61.

21. Debette S, Beiser A, DeCarli C, Au R, Himali JJ, et al. (2010) Association of MRI markers of vascular brain injury with incident stroke, mild cognitive impairment, dementia, and mortality: The framingham offspring study. Stroke 41(4): 600-606.

22. Kissela B, Lindsell CJ, Kleindorfer D, Alwell K, Moomaw CJ, et al. (2009) Clinical prediction of functional outcome after ischemic stroke: The surprising importance of periventricular white matter disease and race. Stroke 40(2): 530-536

23. Tang WK, Chen YK, Liang HJ, Chu CW, Mok CT, et al. (2014) Subcortical white matter infarcts predict 1-year outcome of fatigue in stroke. BMC Neurology 14: 234.

24. Shan ZY, Kwiatek R, Burnet R (2016) Progressive brain changes in patients with chronic fatigue syndrome: A longitudinal MRI study. J Magn Reson Imaging 44(5): 1301-1311.

25. Tseng BY, Gajewski BJ, Kluding PM (2010) Reliability, responsiveness and validity of the visual analog fatigue scale to measure exertion fatigue in people with chronic stroke: a preliminary study. Stroke Res Treat 2010. pii: 412964. doi: 10.4061/2010/412964

26. Krupp LB, LaRocca NG, Muir-Nash J, Steinberg AD (1989) The fatigue severity scale. Application to patients with multiple sclerosis and systemic lupus erythematosus. Arch Neurol 46(10): 1121-1123.

27. Fugl-Meyer AR, Jaasko L, Leyman I, Olsson S, Steglind S (1975) The poststroke hemiplegic patient. 1. a method for evaluation of physical performance. Scand J Rehabil Med 7(1): 13-31.

28. Shelton FD, Volpe BT, Reding M (2001) Motor impairment as a predictor of functional recovery and guide to rehabilitation treatment after stroke. Neurorehabil Neural Repair 15(3): 229 -237. 
29. Meyer TJ, Miller ML, Metzger RL, Borkovec TD (1990) Development and validation of the penn state worry questionnaire. Behav Res Ther 28(6): 487-495.
30. Ann Bowling (2009) The psychometric properties of the older people's quality of life questionnaire, compared with the CASP-19 and the WHOQOL-OLD. Current Gerontology and Geriatrics Research 2009: 12.
(C) Creative Commons Attribution 4.0 International License

For possible submissions Click Here

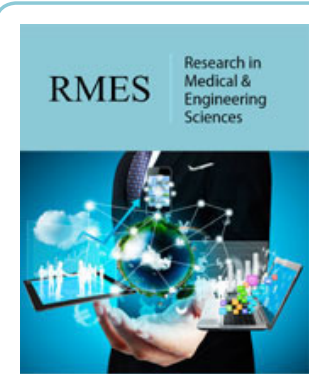

Research in Medical \& Engineering Sciences

\section{Benefits of Publishing with us}

- High-level peer review and editorial services

- Freely accessible online immediately upon publication

- Authors retain the copyright to their work

- Licensing it under a Creative Commons license

- Visibility through different online platforms 